\title{
Sutura meniscal microinvasiva com agulha descartável*
}

\section{Microinvasive Meniscal Suture with Disposable Needle}

Edmar Stieven Filho ${ }^{10}$ Carolline Popovicz Nunes ${ }^{20}$ Fernando Martins Rosa ${ }^{3(0)}$

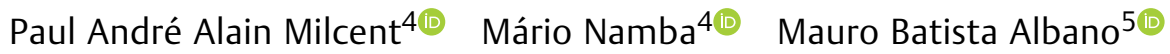

1 Departamento de Cirurgia, Universidade Federal do Paraná,

Endereço para correspondência Edmar Stieven Filho, PhD, Rua Gen.

Curitiba, PR, Brasil

${ }^{2}$ Departamento de Ortopedia e Traumatologia, Hospital das Clínicas, Carneiro, 181, Departamento de Cirurgia, Curitiba, Paraná, Brasil

Universidade Federal do Paraná, Curitiba, Paraná, Brasil

${ }^{3}$ Departamento de Cirurgia, Universidade Federal do Paraná,

Curitiba, PR, Brasil

${ }^{4}$ Departamento de Cirurgia, Universidade Federal do Paraná,

Curitiba, PR, Brasil

${ }^{5}$ Hospital de Clínicas da Universidade Federal do Paraná, Curitiba, PR, Brasil (e-mail: filho2000@gmail.com).

Rev Bras Ortop 2022;57(3):524-528.

\section{Resumo \\ Palavras-chave \\ - lesões do menisco tibial \\ - técnicas de sutura \\ - artroscopia}

A primeira sutura meniscal foi realizada em 1885 e levou cerca de um século para tornar-se popular. Atualmente, os dispositivos de reparo meniscal all-inside são amplamente utilizados. Contudo, esta técnica apresenta a desvantagem de ser um método dependente de dispositivos específicos, apresentando um custo superior aos de outras técnicas. Este valor elevado limita o uso de tal técnica em muitos locais. O objetivo da presente nota técnica é descrever uma técnica de sutura meniscal microinvasiva, como uma modificação da técnica all-inside, utilizando uma agulha descartável de procedimento de $40 \times 12 \mathrm{~mm}$. Os autores acreditam que a modificação proposta para a técnica pode torná-la mais popular, possibilitando o uso da técnica microinvasiva em locais com recursos limitados.

The first meniscal suture was performed in 1885 and took about a century to become popular. Currently, all-inside meniscal repair devices are widely used. However, this technique presents the disadvantage of being a method dependent on specific devices, presenting a higher cost than other techniques. This high cost limits the use of such a technique in many locations. The objective of the present technical note is to describe a microinvasive meniscal suture technique as a modification of the all-inside technique, using a disposable $40 \times 12 \mathrm{~mm}$ procedure needle. The authors believe that the proposed modification to the technique can make it more popular, enabling the use of the microinvasive technique in places with limited resources.

\footnotetext{
* Trabalho realizado no Hospital de Clínicas da Universidade Federal do Paraná, Curitiba, PR, Brasil.
}

recebido

07 de Abril de 2020

aceito

01 de Dezembro de 2020

published online

Outubro 14, 2021
DOI https://doi.org/

$10.1055 / \mathrm{s}-0041-1729938$

ISSN 0102-3616.

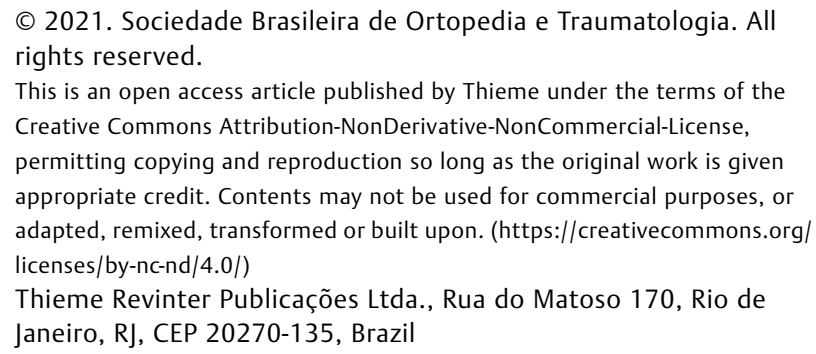

(c) 2021. Sociedade Brasileira de Ortopedia e Traumatologia. All rights reserved.

This is an open access article published by Thieme under the terms of the Creative Commons Attribution-NonDerivative-NonCommercial-License, permitting copying and reproduction so long as the original work is given appropriate credit. Contents may not be used for commercial purposes, or adapted, remixed, transformed or built upon. (https://creativecommons.org/ licenses/by-nc-nd/4.0/) Thieme Revinter Publicações Ltda., Rua do Matoso 170, Rio de Janeiro, RJ, CEP 20270-135, Brazil 


\section{Introdução}

Por muito tempo, o menisco foi considerado uma estrutura sem função que deveria ser removida em qualquer sinal de anormalidade. ${ }^{1}$ Assim, até a década de 1970 , a retirada dos meniscos ocorreu como tratamento padrão. ${ }^{1}$ Contudo, as suturas meniscais demonstram efeito condroprotetor em relação às meniscectomias a longo prazo. ${ }^{2}$ Além disso, diversos estudos mostraram que os meniscos têm papel fundamental na distribuição de carga e estabilidade do joelho; assim, os meniscos são atualmente considerados estruturas vitais no joelho saudável que devem ser preservadas sempre que possível. ${ }^{2}$

A primeira sutura meniscal foi realizada em 1885 por Annandale e levou cerca de um século para tornar-se popular. $^{3}$ É só na década de 1970 , Ikeuchi apud Zhang et al. ${ }^{3}$ descreveu o reparo meniscal por artroscopia, utilizando uma agulha descartável. Com o passar dos anos, houve desenvolvimento de técnicas artroscópicas e de instrumentais, e o procedimento foi então popularizado, ocorrendo um pico de crescimento no número de reparos meniscais entre os anos de 2005 e $2011 .^{3}$

As técnicas de reparo meniscal podem ser divididas em três formas. Elas podem ser realizadas abordando o menisco intra-articular e transpassando o material de sutura para extra-articular (inside-out). Outra forma é iniciar pela pele, introduzindo o material de sutura para intra-articular (outside-in). Ainda existe a técnica all-inside, na qual todos os passos são confeccionados dentro da articulação.

A técnica de sutura artroscópica all-inside apresenta a vantagem de não precisar de incisões na pele, de ter baixa taxa de complicações e resultados semelhantes às outras técnicas de sutura. Uma desvantagem do sistema all-inside é que ele tem um custo maior. Normalmente, são utilizados dispositivos descartáveis, e seu uso eleva significativamente o valor final do procedimento. Isso pode ser um fator limitante do seu uso em muitos locais. ${ }^{4}$

Neste contexto, o objetivo do presente trabalho é descrever uma técnica de sutura meniscal microinvasiva, como uma modificação da técnica all-inside, utilizando uma agulha descartável de procedimento de $40 \times 12 \mathrm{~mm}$.

\section{Descrição da Técnica Cirúrgica}

Inicia-se o procedimento com incisões para o portal anterolateral e anteromedial da artroscopia, adjacentes às bordas lateral e medial do ligamento patelar, respectivamente. Procede-se com a inspeção artroscópica do joelho. A lesão meniscal é identificada e avaliada para determinar sua localização, tamanho e grau de instabilidade. Realiza-se então o preparo da lesão meniscal com debridamento das bordas da lesão para aumentar o potencial de cicatrização. A lesão, então, é reduzida e os pontos são iniciados.

$O$ procedimento é iniciado com a inserção de um fio de ácido poliglicólico 1-0 na agulha $40 \times 12 \mathrm{~mm}$. A agulha é então introduzida na linha interarticular do joelho, com transfixação por via percutânea, de fora para dentro, monitorada pelo lado articular sob artroscopia, transfixando o menisco, na região lesionada (- Figura $\mathbf{1}$ ).

A agulha conduz um fio de sutura que permanecerá no trajeto, e com a ajuda de um Grasper introduzido pelo portal anteromedial, o fio intra-articular é puxado pelo portal, mantendo o restante do fio dentro da agulha. A agulha é então retirada do menisco sem, contudo, retirá-la da pele (-Figura 2).

Depois, a agulha é reintroduzida no menisco próxima da primeira inserção (- Figura 3).

A segunda extremidade do fio é então puxada com o auxílio do Grasper ( - Figura 4). Com as duas extremidades do fio em mãos, o cirurgião pode realizar o nó fora da articulação. Após a confecção do nó, ele é conduzido para a região desejada com auxílio de um empurrador de nó (-Figura 5).

\section{Comentários Finais}

As cirurgias meniscais estão entre os procedimentos mais comumente executados em ortopedia. ${ }^{4}$ As opções primárias atuais para cirurgia meniscal artroscópica são meniscectomia parcial ou reparo meniscal. Porém, as descobertas sobre as funções vitais do menisco e o desenvolvimento de osteoartrite relatada após sua ressecção obrigaram os cirurgiões a proteger o máximo possível dessa estrutura, reparando ou reconstruindo o menisco sempre que possivel. ${ }^{2}$ Atualmente,
JOELHO DIREITO

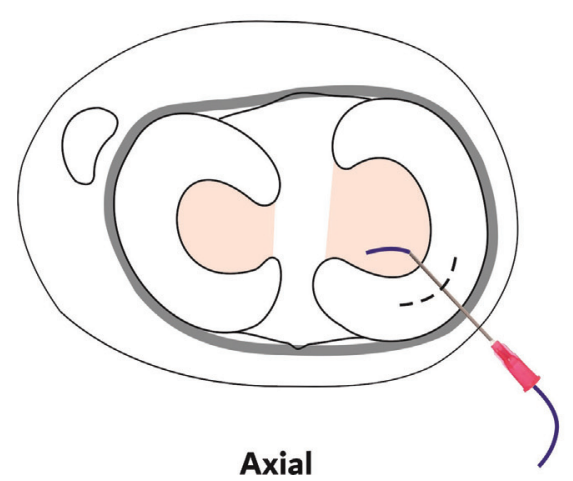

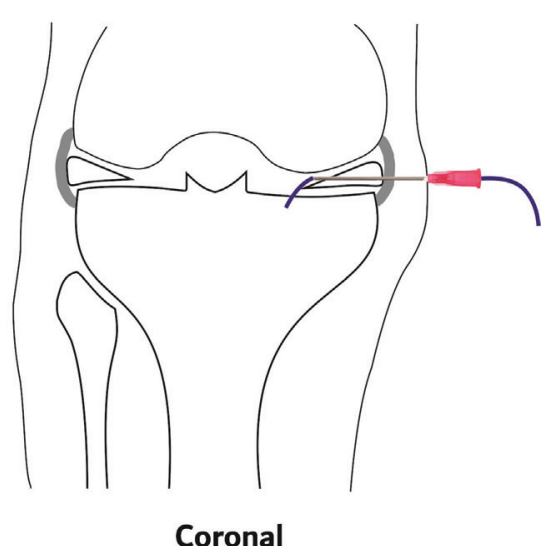

Fig. 1 Visão axial e sagital da inserção da agulha com o fio, na linha interarticular do joelho, transfixando a região lesionada. 

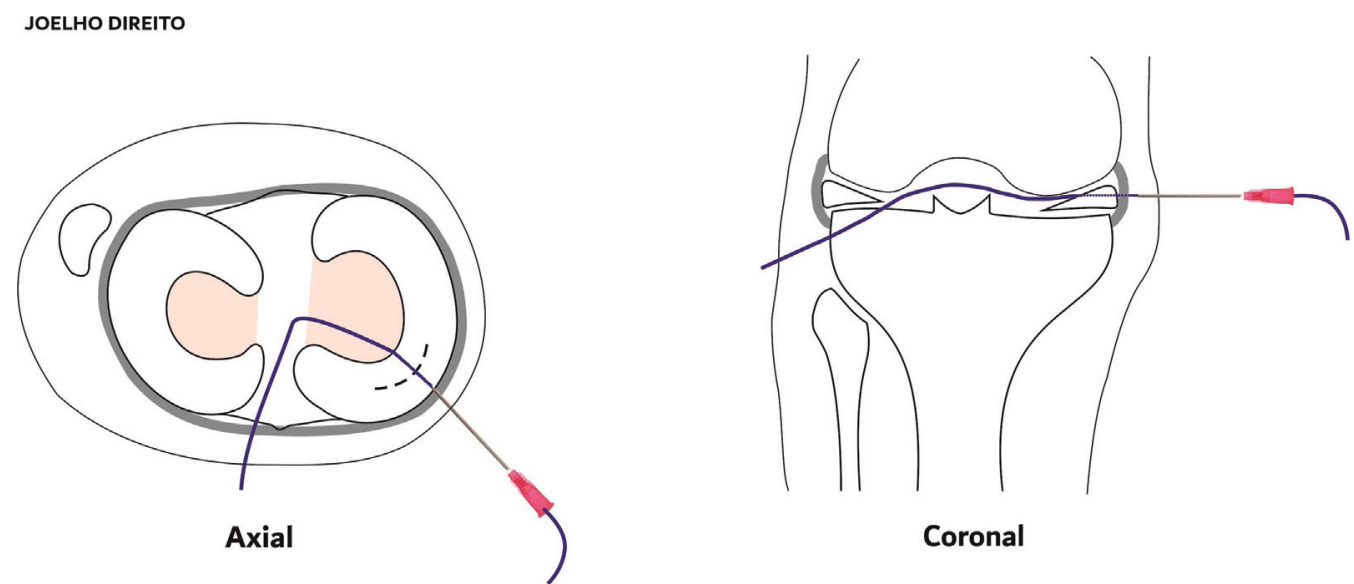

Coronal

Fig. 2 Visão axial e sagital da retirada da agulha do menisco, parando entre a cápsula e a pele.
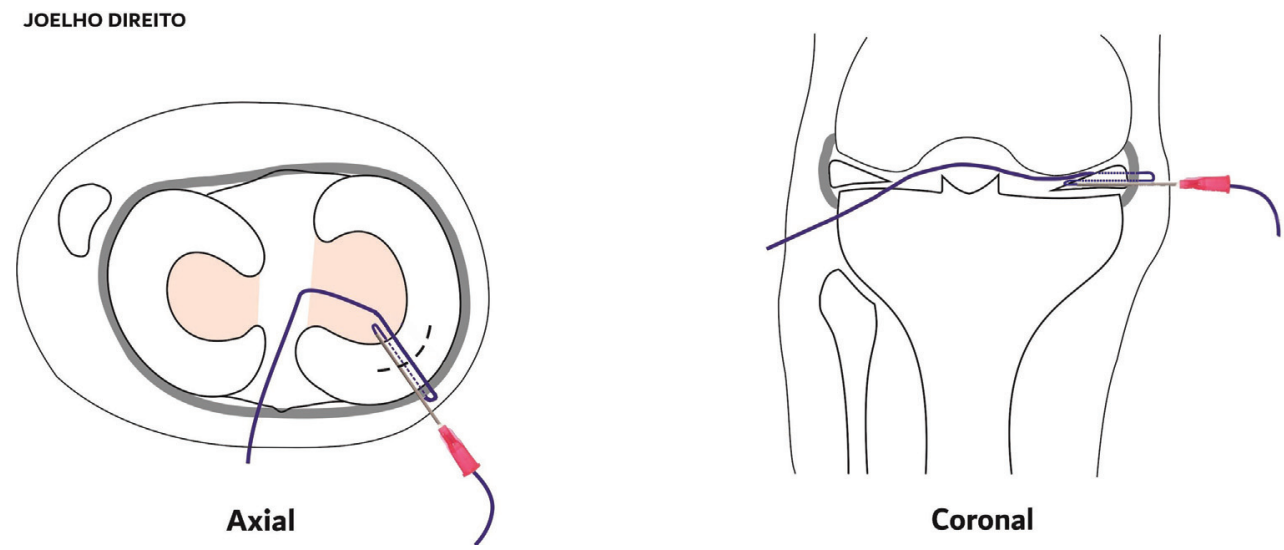

Coronal

Fig. 3 Visão axial e sagital da reintrodução da agulha e fio no menisco, próximos à primeira inserção.

\section{JOELHO DIREITO}

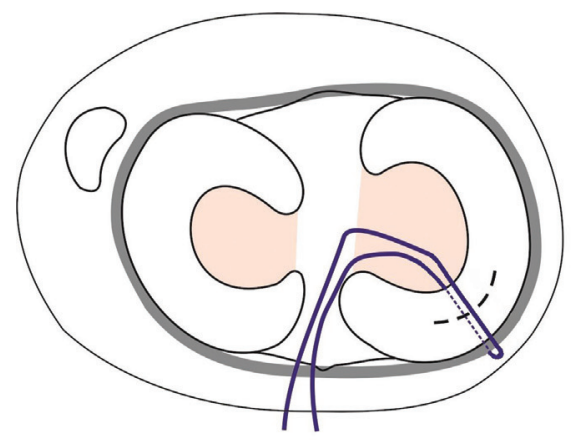

Axial

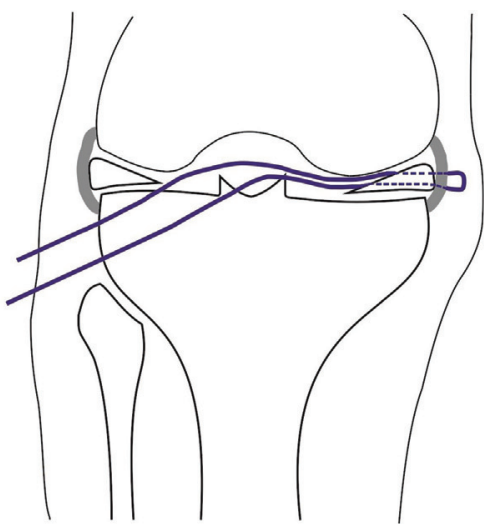

Coronal

Fig. 4 Visão axial e sagital das extremidades do fio fora da articulação.

existem três técnicas principais para o reparo do menisco: inside-out, outside-in e all-inside. Os dispositivos de reparo meniscal all-inside, relatados pela primeira vez em 1993, são amplamente utilizados atualmente. ${ }^{4}$ Contudo, essa técnica apresenta a desvantagem de ser um método dependente de dispositivos específicos, desenvolvidos apenas por poucas empresas; consequentemente, tais dispositivos apresentam um custo superior aos de outras técnicas. ${ }^{4}$ Nesse cenário, os autores apresentam uma modificação da técnica de sutura all-inside utilizando uma agulha descartável de procedimento de $40 \times 12 \mathrm{~mm}$. A variação dá-se no instrumental utilizado, que viabiliza o emprego da técnica de maneira mais democrática em virtude do baixo custo e da disponibilidade dos materiais utilizados.

Um reparo bem-sucedido requer estabilização do tecido meniscal lesionado durante o processo de cicatrização; neste 


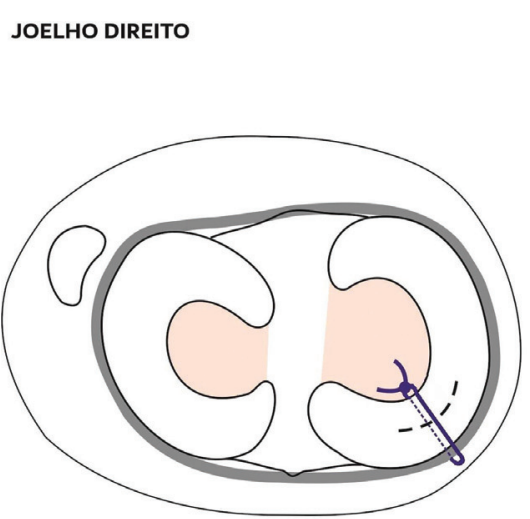

Axial

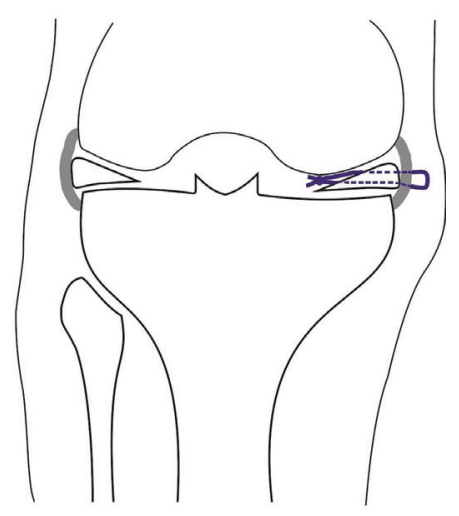

Coronal

Fig. 5 Visão axial e sagital do resultado final da confecção do nó.

processo, muitos fatores podem influenciar no resultado final, incluindo a escolha da técnica de reparo, métodos de reparo e número de suturas. ${ }^{5}$ Embora existam várias técnicas de reparo meniscal disponíveis, a técnica outside-in ainda é considerada padrão ouro para reparo meniscal. No entanto, a técnica all-inside tem a vantagem de não exigir uma incisão adicional, levando a uma diminuição no tempo operatório. ${ }^{4}$ Além disso, em uma recente revisão sistemática de Fillingham, quando comparadas as técnicas de reparo all-inside e outside-in, não foram observadas diferenças nas taxas de falha, nos resultados funcionais ou nas taxas de complicações. ${ }^{6}$ Assim, a técnica all-inside tem sido um método cada vez mais usado para a maioria das lesões meniscais devido às suas vantagens de evitar a abertura de portais acessórios e incisões adicionais, fácil aplicabilidade, uso de implantes bioabsorvíveis e risco relativamente menor de lesões nas estruturas neurovasculares posteriores. ${ }^{4}$

A escolha da técnica a ser utilizada para o reparo meniscal depende de diversos fatores, como local da lesão, tipo, etiologia, tempo, associação com lesão ligamentar, expectativas e idade do paciente, além da experiência do cirurgião com a técnica a ser utilizada. Os autores acreditam que a técnica de sutura meniscal microinvasiva seja mais indicada para lesões longitudinais do corpo e corno anterior. Em contrapartida, os autores não indicam o uso da técnica para lesões próximas a estruturas nobres, pois o risco de lesão inadvertida dessas estruturas é alto para qualquer técnica percutânea.

Embora seja uma técnica promissora, a modificação da técnica all-inside proposta pelos autores apresenta algumas dificuldades. Uma delas é acertar o local da lesão, já que o procedimento inicia com a agulha na pele e segue em direção ao menisco. Outro ponto importante é não ter o movimento bloqueado por contato da agulha com o platô tibial ou com o côndilo femoral. Além disso, é necessário que o cirurgião tenha habilidade de realizar o ponto extra-articular e conduzi-lo ao menisco. Isso pode ser um fator limitante, uma vez que tal habilidade não é comum aos cirurgiões do joelho, sendo mais praticada em cirurgias artroscópicas do ombro. Pelos motivos acima, suturas realizadas com dispositivos de sutura all-inside tendem a ser mais rápidas do que a sutura realizada de maneira manual, como a proposta pelo presente estudo.

De maneira geral, nas últimas décadas, observou-se na área médica um aumento dos custos dos tratamentos em função da introdução de tecnologias mais modernas e novos medicamentos. ${ }^{7}$ Isso acaba tornando-se um problema para os sistemas de saúde públicos universais, como o Sistema Único de Saúde (SUS) e os convênios privados de saúde, onde soma-se a falta de recursos ou o surgimento dessas novas tecnologias, dificultando ainda mais a gestão da saúde no país. ${ }^{7}$ Neste contexto, há uma tendência mundial pela busca de soluções eficientes que possibilitem o uso de novas abordagens que podem beneficiar o paciente associadas ao controle dos gastos. ${ }^{8}$ Por exemplo, atualmente, há dificuldade de liberação dos dispositivos de sutura meniscal allinside nos convênios e no SUS, já que eles encarecem o preço final da cirurgia. Isso limita o uso da técnica, mesmo quando seria a melhor opção de abordagem para o paciente. Os autores acreditam que a modificação proposta para a técnica pode torná-la mais popular, possibilitando o uso da técnica microinvasiva em locais com recursos limitados.

\section{Suporte Financeiro}

Não houve suporte financeiro de fontes públicas, comerciais, ou sem fins lucrativos.

\section{Conflito de Interesses}

Os autores declaram não haver conflito de interesses.

\section{Referências}

1 Dandy DJ, Jackson RW. The diagnosis of problems after meniscectomy. J Bone Joint Surg Br 1975;57(03):349-352

$2 \mathrm{Xu} \mathrm{C}$, Zhao J. A meta-analysis comparing meniscal repair with meniscectomy in the treatment of meniscal tears: the more meniscus, the better outcome? Knee Surg Sports Traumatol Arthrosc 2015;23(01):164-170

3 Zhang AL, Miller SL, Coughlin DG, Lotz JC, Feeley BT. Tibiofemoral contact pressures in radial tears of the meniscus treated with allinside repair, inside-out repair and partial meniscectomy. Knee 2015;22(05):400-404 
4 Abrams GD, Frank RM, Gupta AK, Harris JD, McCormick FM, Cole $\mathrm{BJ}$. Trends in meniscus repair and meniscectomy in the United States, 2005-2011. Am J Sports Med 2013;41(10):2333-2339

5 Beaufils P, Pujol N. Meniscal repair: Technique. Orthop Traumatol Surg Res 2018;104(1S)S137-S145

6 Fillingham YA, Riboh JC, Erickson BJ, Bach BR Jr, Yanke AB. Insideout versus all-inside repair of isolated meniscal tears. An updated systematic review. Am J Sports Med 2017;45(01):234-242
7 Souza AA, Guerra M, Avelar EA. Proposta de metodologia para a implantação do sistema de custeio baseado em atividades para organizações hospitalares. In: Paper presented at: XVI Congresso Brasileiro De Custos, 2009, Fortaleza, CE. 2009

8 Silva JLV, Namba MM, Pereira FA, et al. Sutura meniscal “insideout" com agulha de anestesia peridural. Rev Bras Ortop 2004;39 (05):264-269 